\title{
Multinucleon transfer reaction in time-dependent Hartree-Fock theory
}

\author{
Kazuyuki Sekizawa ${ }^{1,2}$ and Kazuhiro Yabana ${ }^{2,3}$ \\ ${ }^{1}$ Faculty of Physics, Warsaw University of Technology, ulica Koszykowa 75, 00-662 Warsaw, \\ Poland \\ ${ }^{2}$ Graduate School of Pure and Applied Sciences, University of Tsukuba, Tsukuba 305-8571, \\ Japan \\ ${ }^{3}$ Center for Computational Sciences, University of Tsukuba, Tsukuba 305-8577, Japan \\ E-mail: sekizawa@if.pw.edu.pl, yabana@nucl.ph.tsukuba.ac.jp
}

\begin{abstract}
Time-dependent Hartree-Fock (TDHF) theory has achieved a remarkable success in describing and understanding nuclear many-body dynamics from nucleons' degrees of freedom. We here report our investigation of multinucleon transfer (MNT) processes employing the TDHF theory. To calculate transfer probabilities for channels specified by the number of protons and neutrons included in reaction products, a particle-number projection (PNP) method has been developed. The PNP method is also used to calculate excitation energies of reaction products. Combined use of the PNP method with a statistical model, we can evaluate MNT cross sections taking account of effects of particle evaporation. Using these methods, we evaluate MNT cross sections for ${ }^{40,48} \mathrm{Ca}+{ }^{124} \mathrm{Sn},{ }^{40} \mathrm{Ca}+{ }^{208} \mathrm{~Pb}$, and ${ }^{58} \mathrm{Ni}+{ }^{208} \mathrm{~Pb}$ reactions. From systematic analyses, we find that cross sections for channels with a large reaction probability are in good agreement with experimental data. However, the agreement becomes less accurate as the number of transferred nucleons increases. Possible directions to improve the description are discussed.
\end{abstract}

keywords: TDHF, particle-number projection, multinucleon transfer reaction

\section{Introduction}

In low-energy heavy ion reactions at around the Coulomb barrier, a variety of phenomena reflecting quantum many-body dynamics are observed. In subbarrier reactions, fusion and transfer as well as quasi-elastic processes take place. The subbarrier fusion process can be regarded as quantum many-body tunneling phenomena between two colliding nuclei. In reactions close to the barrier, a neck is formed between them, providing a pathway of nucleon exchange between the projectile and the target nuclei. The nucleon exchange causes an energy dissipation, a transfer of energy from relative motion of the projectile and the target nuclei into their internal excitations. While fusion reactions are commonly observed in low-energy reactions, they are hindered in heavy systems by the appearance of quasifission processes. Reactions involving superfluid nuclei attract another interest, effects of pairing correlations on nuclear dynamics.

We have been working on multinucleon transfer (MNT) processes, one of phenomena that attracted substantial interests in low-energy heavy ion reactions. The MNT processes become dominant in reactions at energies around and above the Coulomb barrier. Besides curiosity in their reaction mechanisms, the MNT processes have been attracting interests as a new means to produce unstable nuclei whose production is difficult by other methods $[1,2,3,4,5,6,7]$. For example, it has been discussed to use the MNT reactions to produce neutron-rich nuclei around the neutron magic number $N=126[8,9]$, which are considered to play a significant role in producing the third peak structure in the solar abundance. 
In the past, theoretical investigations on MNT processes have been extensively made using semiclassical models such as GRAZING [10], complex WKB (CWKB) [11], a dynamical model based on Langevin-type equations of motion [12, 13], and dinuclear system model (DNS) $[14,15,16,17]$. The semiclassical models of GRAZING and CWKB have been quite successful to describe MNT processes in peripheral collisions (for a review, see Ref. [18]). However, it has turned out that GRAZING substantially underestimates cross sections associated with damped collisions forming reaction products which have largely different masses from initial ones $[7,19]$. This is natural because reactions at a small impact parameter region inside the nuclear rainbow are not considered in the GRAZING calculations. The dynamical Langevin model and DNS have been successful in describing not only peripheral collisions but also damped collisions such as deep inelastic collisions, quasifission, fusionfission, and fusion reactions. Although the dynamical Langevin model is recognized as one of the most promising methods to examine MNT processes including a damped collision, the model contains some empirical parameters [4] that restrict their predictive power. To further extend our understanding on underlying reaction mechanisms and to improve reliability to predict cross sections, it is highly desired to develop a microscopic framework for the MNT reaction. To this end, we have investigated MNT processes employing the time-dependent Hartree-Fock (TDHF) theory.

As described in detail in other chapters, the TDHF theory has been quite successful to study nuclear dynamics. Starting from 1970s, substantial efforts have been devoted for developing computational and analytical methods, and for applications (for a review, see Refs. $[3,20])$. It has been considered that the TDHF theory is suitable to describe quantities associated with one-body operators [3], and has been applied to averaged quantities in most applications. To describe MNT cross sections, we go beyond it, extracting transfer probabilities from the TDHF wave function after collision for each channel specified by the nucleon numbers in the reaction products.

In 1977, S.E. Koonin et al. proposed a method to calculate transfer probabilities from a TDHF wave function after collision [21]. In 1983, the same method was applied to electron transfer processes in atomic collisions by H.J. Lüde and R.M. Dreizler [22]. The method was also applied to a collision of a highly-charged ion and an atom [23]. Although the method was useful to investigate transfer processes, applications were limited to relatively small systems because of computational limitations. The method requires calculations of determinants of $N$-dimensional matrices many times, where $N$ stands for the number of particles in the system. If we calculate probabilities for all possible processes, the number of calculations of the determinants becomes $2^{N}$. Thus the method can be applied to systems less than a few tens of nucleons. In 2011, C. Simenel proposed an efficient method [24] to calculate the transfer probabilities using the particle-number projection (PNP) method. The PNP method succeeds to reduce the computational costs significantly, while being analytically equivalent to the method proposed in the past. The number of calculations of determinants necessary to carry out the PNP is about at most several hundreds and is independent of the number of particles in the system. Using the PNP method, it has become possible to calculate the transfer probabilities for reactions involving heavy nuclei.

In this chapter, we will report results of our TDHF calculations [25] for the MNT reactions of ${ }^{40} \mathrm{Ca}+{ }^{124} \mathrm{Sn}$ at $E_{\text {lab }}=170 \mathrm{MeV},{ }^{48} \mathrm{Ca}+{ }^{124} \mathrm{Sn}$ at $E_{\text {lab }}=174 \mathrm{MeV},{ }^{40} \mathrm{Ca}+{ }^{208} \mathrm{~Pb}$ at $E_{\text {lab }}=235$ and $249 \mathrm{MeV}$, and ${ }^{58} \mathrm{Ni}+{ }^{208} \mathrm{~Pb}$ at $E_{\text {lab }}=328.4 \mathrm{MeV}$, for which precise experimental data are available $[26,27,28,29]$. We consider that comparisons between theory and measurements for those systems with different $N / Z$ ratios and different charge product $Z_{\mathrm{P}} Z_{\mathrm{T}}$ will provide useful information on reaction dynamics [25].

It has been well known that a fast charge equilibration process takes place in transfer reactions at low-energy where strength of the driving force for the charge equilibration process is related to the difference of $N / Z$ ratio between projectile and target nuclei. While the ${ }^{48} \mathrm{Ca}+{ }^{124} \mathrm{Sn}$ system has a small $N / Z$ asymmetry, all other systems have relatively large $N / Z$ asymmetries. It has also been well known that a product of charges of projectile and target nuclei, $Z_{\mathrm{P}} Z_{\mathrm{T}}$, determines qualitative aspects of low-energy heavy ion reactions. When the charge product $Z_{\mathrm{P}} Z_{\mathrm{T}}$ exceeds a critical value of about 1600 , occurrence of fusion reactions is substantially suppressed. The four systems examined have values of 1000 for 
${ }^{40,48} \mathrm{Ca}+{ }^{124} \mathrm{Sn}, 1640$ for ${ }^{40} \mathrm{Ca}+{ }^{208} \mathrm{~Pb}$, and 2296 for ${ }^{58} \mathrm{Ni}+{ }^{208} \mathrm{~Pb}$. Since the values are below, around, and above the critical value, respectively, we expect that different reaction dynamics will be seen in these systems.

Fragment nuclei produced after MNT processes are expected to be highly excited. Measurements are carried out for fragments which are deexcited by emissions of particles and photons. To compare calculated cross sections with measurements, proper treatments of deexcitation processes such as nucleon evaporation and transfer induced fission are required. Because these secondary processes take place in a much longer timescale than that can be simulated by direct time evolution calculations, we should estimate the number of nucleons to be evaporated from an excited reaction product by a method other than the direct TDHF calculations. We will use a statistical model for that purpose. As an input for such statistical calculations, excitation energy of a reaction product in each transfer channel is required. Recently, we invented a method to calculate the excitation energy using the PNP method [30]. We review our theoretical framework, present calculated cross sections including the particle evaporation effect, and compare them with experimental data.

This chapter is organized as follows. In Sec. 2, we present theoretical framework and numerical setting of our approach putting emphasis on the PNP method applied for TDHF wave functions. In Sec. 3, we show results of our TDHF calculations for MNT processes in ${ }^{40,48} \mathrm{Ca}+{ }^{124} \mathrm{Sn},{ }^{40} \mathrm{Ca}+{ }^{208} \mathrm{~Pb}$, and ${ }^{58} \mathrm{Ni}+{ }^{208} \mathrm{~Pb}$ reactions, for which precise experimental data are available. Effects of particle evaporation on the cross sections are investigated employing a simple statistical model. In Sec. 4, a summary and a future prospect are presented.

\section{Theoretical formalism}

\subsection{TDHF wave function for heavy ion reactions}

We start with considering a TDHF wave function for low-energy heavy ion reactions. We denote the number of nucleons of the projectile and target nuclei as $N_{\mathrm{A}}$ and $N_{\mathrm{B}}$, respectively. The total number of nucleons is expressed as $N=N_{\mathrm{A}}+N_{\mathrm{B}}$. In TDHF calculations, the many-body wave function of the system is expressed by a single Slater determinant,

$$
\Phi\left(\mathbf{r}_{1} \sigma_{1}, \cdots, \mathbf{r}_{N} \sigma_{N}, t\right)=\frac{1}{\sqrt{N !}} \operatorname{det}\left\{\phi_{i}\left(\mathbf{r}_{j} \sigma_{j}, t\right)\right\}
$$

where $\phi_{i}(\mathbf{r} \sigma, t)$ denotes single-particle wave functions of nucleons where $\sigma$ denotes the spin coordinate. In this Section, we omit isospin degree of freedom for simplicity.

The initial TDHF wave function before the reaction is prepared as a product of two Slater determinants which are the static Hartree-Fock (HF) solutions of projectile and target nuclei, boosted with velocities determined by the initial condition of relative motion. We divide the space into two subspaces referring to the position of two nuclei and define number operators for the two subspaces, $\hat{N}_{\mathrm{A}}$ and $\hat{N}_{\mathrm{B}}$, by

$$
\hat{N}_{\tau}=\int \Theta_{\tau}(\mathbf{r}) \sum_{i=1}^{N} \delta\left(\mathbf{r}-\mathbf{r}_{i}\right) d \mathbf{r}=\sum_{i=1}^{N} \Theta_{\tau}\left(\mathbf{r}_{i}\right),
$$

where $\Theta_{\tau}(\mathbf{r})$ denotes a space division function defined by

$$
\Theta_{\tau}(\mathbf{r})= \begin{cases}0 & \text { for } \mathbf{r} \in \tau \\ 1 & \text { for } \mathbf{r} \notin \tau\end{cases}
$$

The initial TDHF wave function is apparently an eigenstate of $\hat{N}_{\tau}$,

$$
\hat{N}_{\tau}|\Phi(t=0)\rangle=N_{\tau}|\Phi(t=0)\rangle,
$$

where $\tau$ refers to a subspace either $A$ or $B$. Here and hereafter, we often use a bracket notation, e.g. $|\Phi(t)\rangle \equiv \Phi\left(\mathbf{r}_{1} \sigma_{1}, \cdots, \mathbf{r}_{N} \sigma_{N}, t\right)$, to simplify notations.

Evolving orbital wave functions by solving the TDHF equation, two nuclei approach to each other. Each single-particle wave function, which initially belongs to either projectile 
or target nucleus, starts to extend into the other nucleus. We consider cases where binary fragments, a projectile-like fragment (PLF) and a target-like fragment (TLF), are produced after the reaction. At a certain time $t=t_{\mathrm{f}}$ at which the binary reaction products are spatially separated, we again divide the space into two, $A^{\prime}$ that includes the PLF and $B^{\prime}$ that includes the TLF. We define the number operators for the subspaces $A^{\prime}$ and $B^{\prime}$ as $\hat{N}_{\mathrm{A}^{\prime}}$ and $\hat{N}_{\mathrm{B}^{\prime}}$, respectively. The TDHF wave function after collision is generally not an eigenstate of those operators:

$$
\hat{N}_{\tau}\left|\Phi\left(t=t_{\mathrm{f}}\right)\right\rangle \neq N_{\tau}\left|\Phi\left(t=t_{\mathrm{f}}\right)\right\rangle
$$

where $\tau=A^{\prime}$ or $B^{\prime}$.

In the following, we denote the TDHF wave function after collision at time $t=t_{\mathrm{f}}$ as $|\Psi\rangle \equiv\left|\Phi\left(t=t_{\mathrm{f}}\right)\right\rangle$ omitting the time index. We also denote single-particle wave functions at $t=t_{f}$ as $\psi_{i}(\mathbf{r} \sigma)$. We omit a prime from $A^{\prime}$ and $B^{\prime}$ without confusion.

\subsection{Particle-number projection method}

As described above, the TDHF wave function after collision, $|\Psi\rangle$, is not an eigenstate of the operator $\hat{N}_{\tau}$ but a superposition of states with different mass partitions:

$$
|\Psi\rangle=\sum_{n=0}^{N}\left|\Psi_{n}\right\rangle,
$$

where $\left|\Psi_{n}\right\rangle$ denotes a state in which $n$ nucleons are in the spatial region $A$ and $N-n$ nucleons are in the spatial region $B$.

The PNP operator is used to extract a component of a given number of nucleons from $|\Psi\rangle$. It is given by $[24,25]$

$$
\begin{aligned}
\hat{P}_{n} & =\sum_{\left\{\tau: A^{n} B^{N-n}\right\}} \Theta_{\tau_{1}}\left(\mathbf{r}_{1}\right) \cdots \Theta_{\tau_{N}}\left(\mathbf{r}_{N}\right) \\
& =\frac{1}{2 \pi} \int_{0}^{2 \pi} e^{i\left(n-\hat{N}_{A}\right) \theta} d \theta .
\end{aligned}
$$

The $\left\{\tau: A^{n} B^{N-n}\right\}$ under the summation in Eq. (7) means that the sum is taken for all possible combinations where $A$ appears $n$ times and $B$ appears $N-n$ times in the sequence of $\tau_{1} \tau_{2} \ldots \tau_{N}$.

Using the PNP operator, the state $\left|\Psi_{n}\right\rangle$ in Eq. (6) can be expressed as $\left|\Psi_{n}\right\rangle=\hat{P}_{n}|\Psi\rangle$. The probability $P_{n}$ that $n$ nucleons are in the spatial region $A$ and the other $N-n$ nucleons are in the spatial region $B$ is given by

$$
P_{n}=\left\langle\Psi_{n} \mid \Psi_{n}\right\rangle=\left\langle\Psi\left|\hat{P}_{n}\right| \Psi\right\rangle .
$$

When we substitute the PNP operator of the form of Eq. (7) into Eq. (9), we get the formula,

$$
P_{n}=\sum_{\left\{\tau: A^{n} B^{N-n}\right\}} \operatorname{det}\left\{\left\langle\psi_{i} \mid \psi_{j}\right\rangle_{\tau_{j}}\right\},
$$

where $\left\langle\psi_{i} \mid \psi_{j}\right\rangle_{\tau} \equiv \sum_{\sigma} \int d \mathbf{r} \Theta_{\tau}(\mathbf{r}) \psi_{i}^{*}(\mathbf{r} \sigma) \psi_{j}(\mathbf{r} \sigma)$. To calculate $P_{n}$ using this formula, determinants of the matrix $\left\langle\psi_{i} \mid \psi_{j}\right\rangle_{\tau}$ need to be calculated as many as ${ }_{N} C_{n}$. Calculations of the determinants become quite difficult once the total number of nucleons in the system, $N$, becomes large.

When we use the PNP operator of the form of Eq. (8), we get alternative formula given by

$$
P_{n}=\frac{1}{2 \pi} \int_{0}^{2 \pi} e^{i n \theta} \operatorname{det} \mathcal{B}(\theta) d \theta,
$$

where $\mathcal{B}(\theta)$ denotes an $N$-dimensional matrix,

$$
\mathcal{B}_{i j}(\theta)=\left\langle\psi_{i} \mid \psi_{j}\right\rangle_{B}+e^{-i \theta}\left\langle\psi_{i} \mid \psi_{j}\right\rangle_{A} .
$$


The integral over the phase $\theta$ in Eq. (11) can be carried out using the trapezoidal rule discretizing the interval into a uniform grid. Using this formula, the number of calculations of the determinants required to obtain $P_{n}$ is the number of discrete points $M$ to carry out the integral numerically, and is independent of the total number of nucleons in the system. We have found that $M=200$ is sufficient for the systems analyzed in this chapter.

\subsection{Expectation values in particle-number projected states}

In the particle-number projected wave function, $\left|\Psi_{n}\right\rangle$, two reaction products are included: a PLF composed of $n$ nucleons inside the spatial region $A$ and a TLF composed of $N-n$ nucleons inside the spatial region $B$. In this subsection, we present a method to calculate expectation values of operators for one of the reaction products included in $\left|\Psi_{n}\right\rangle$ [30].

We first introduce a decomposition of operators according to the spatial region in which the operator acts. For local one-body operators, $\hat{\mathcal{O}}^{(1)}$, it is rather trivial:

$$
\begin{aligned}
\hat{\mathcal{O}}^{(1)} & =\sum_{i=1}^{N}\left[\Theta_{A}\left(\mathbf{r}_{i}\right)+\Theta_{B}\left(\mathbf{r}_{i}\right)\right] \hat{o}^{(1)}\left(\mathbf{r}_{i} \sigma_{i}\right) \\
& =\hat{\mathcal{O}}_{A}^{(1)}+\hat{\mathcal{O}}_{B}^{(1)},
\end{aligned}
$$

where $\hat{O}_{\tau}^{(1)}$ denotes a one-body operator which acts only when a nucleon is in the spatial region $\tau$. For local two-body operators, $\hat{\mathcal{O}}^{(2)}$, we decompose as

$$
\begin{aligned}
\hat{\mathcal{O}}^{(2)}= & \sum_{i<j}^{N}\left[\Theta_{A}\left(\mathbf{r}_{i}\right)+\Theta_{B}\left(\mathbf{r}_{i}\right)\right]\left[\Theta_{A}\left(\mathbf{r}_{j}\right)+\Theta_{B}\left(\mathbf{r}_{j}\right)\right] \hat{o}^{(2)}\left(\mathbf{r}_{i} \sigma_{i}, \mathbf{r}_{j} \sigma_{j}\right) \\
= & \sum_{i<j}^{N}\left[\Theta_{A}\left(\mathbf{r}_{i}\right) \Theta_{A}\left(\mathbf{r}_{j}\right)+\Theta_{B}\left(\mathbf{r}_{i}\right) \Theta_{B}\left(\mathbf{r}_{j}\right)\right. \\
& \left.\quad+\Theta_{A}\left(\mathbf{r}_{i}\right) \Theta_{B}\left(\mathbf{r}_{j}\right)+\Theta_{B}\left(\mathbf{r}_{i}\right) \Theta_{A}\left(\mathbf{r}_{j}\right)\right] \hat{o}^{(2)}\left(\mathbf{r}_{i} \sigma_{i}, \mathbf{r}_{j} \sigma_{j}\right) \\
= & \hat{\mathcal{O}}_{A}^{(2)}+\hat{\mathcal{O}}_{B}^{(2)}+\hat{\mathcal{O}}_{A B}^{(2)},
\end{aligned}
$$

where $\hat{\mathcal{O}}_{\tau}^{(2)}$ denotes a two-body operator which acts only when both two nucleons are in the spatial region $\tau$, while $\hat{\mathcal{O}}_{A B}^{(2)}$ denotes a two-body operator which acts when a nucleon is in the spatial region $A$ and the other nucleon is in the spatial region $B$. We note that, when the PLF and the TLF are separated by more than a distance of nuclear force, only long-ranged Coulomb interaction between protons contribute to $\hat{\mathcal{O}}_{A B}^{(2)}$.

The expectation value of the operator $\hat{\mathcal{O}}$ for the reaction product which is composed of $n$ nucleons and locates inside the spatial region $A$ is given by

$$
\mathcal{O}_{n}^{A}=\frac{\left\langle\Psi_{n}\left|\hat{\mathcal{O}}_{A}\right| \Psi_{n}\right\rangle}{\left\langle\Psi_{n} \mid \Psi_{n}\right\rangle}
$$

Using the PNP operator of the form of Eq. (8), we can evaluate the expectation value of Eq. (15) as

$$
\mathcal{O}_{n}^{A}=\frac{1}{2 \pi P_{n}} \int_{0}^{2 \pi} e^{i n \theta} \operatorname{det} \mathcal{B}(\theta)\left\langle\Psi\left|\hat{\mathcal{O}}_{A}\right| \tilde{\Psi}(\theta)\right\rangle d \theta
$$

where $|\tilde{\Psi}(\theta)\rangle$ denotes a Slater determinant composed of the following single-particle wave functions,

$$
\tilde{\psi}_{i}(\mathbf{r} \sigma, \theta)=\sum_{k=1}^{N} \psi_{k}(\mathbf{r} \sigma)\left(\mathcal{B}^{-1}(\theta)\right)_{k i} .
$$

We note that, because $\left\{\tilde{\psi}_{i}(\theta)\right\}$ are bi-orthonormal to $\left\{\psi_{i}\right\}$, i.e. $\left\langle\psi_{i} \mid \tilde{\psi}_{j}(\theta)\right\rangle=\delta_{i j}$, calculations of matrix elements, $\left\langle\Psi\left|\hat{\mathcal{O}}_{A}\right| \tilde{\Psi}(\theta)\right\rangle$, can be carried out easily. In practical calculations, we need to perform the PNP for both neutrons and protons. 
We use Eq. (16) to evaluate the excitation energy of a reaction product for every transfer channel. As an operator, we consider the Hamiltonian for the reaction product inside the spatial region $A, \hat{H}_{A}=\hat{T}_{A}+\hat{V}_{A}^{\text {Skyrme }}$. Putting it into Eq. (16) (more precisely, using the corresponding formula of the Skyrme energy density functional kernel), we evaluate the energy expectation value of the reaction product composed of $N$ neutrons and $Z$ protons, which we denote as $\mathcal{E}_{N, Z}^{A}$. To remove the energy associated with a translational motion, we make a Galilean transformation for the single-particle wave functions, moving to the rest frame of the reaction product inside the spatial region $A$. The excitation energy of the reaction product is evaluated by subtracting the ground state energy,

$$
E_{N, Z}^{*}(E, b) \equiv \mathcal{E}_{N, Z}^{A}(E, b)-E_{N, Z}^{\mathrm{g} . \mathrm{s} .}
$$

\subsection{MNT cross sections with evaporation effects}

For a given incident energy $E$ and impact parameter $b$, we have a TDHF wave function after collision, $\Psi(E, b)$. We can calculate the probability for a production of a nucleus having $N$ neutrons, $P_{N}(E, b)$, and that having $Z$ protons, $P_{Z}(E, b)$, using Eq. (11). The probability producing the reaction product having $N$ neutrons and $Z$ protons is given by $P_{N, Z}(E, b)=P_{N}(E, b) P_{Z}(E, b)$. Integrating it over the impact parameter, we obtain the corresponding cross section,

$$
\sigma_{N Z}(E)=2 \pi \int_{b_{\min }}^{\infty} b P_{N, Z}(E, b) d b
$$

where $b_{\text {min }}$ denotes the minimum impact parameter at which we observed binary reaction products after collision.

The above formula gives us the MNT cross sections in which the numbers of neutrons and protons correspond to those immediately after the fragments are formed. Reaction products of MNT processes immediately after the separation are highly excited, especially for collisions at small impact parameters. Therefore, a proper evaluation of effects of secondary disintegration processes is necessary. Since these processes take place in a much longer timescale than the timescale producing binary fragment nuclei, we treat the effect by a statistical model.

As a first investigation [31], we have taken a simple statistical model of particle evaporation developed by I. Dostrovsky and his coworkers [32]. In this model, evaporation of neutrons, protons, deuterons, tritons, ${ }^{3} \mathrm{He}$, and ${ }^{4} \mathrm{He}$ are taken into account. As an input, this model only requires the excitation energy of the nucleus, $E^{*}$. Using this model, we evaluate evaporation probabilities, $P_{N, Z}^{n, z}\left[E_{N, Z}^{*}\right]$, emitting $n$ neutrons and $z$ protons from the nucleus composed originally of $N$ neutrons and $Z$ protons with excitation energy $E_{N, Z}^{*}$. The MNT cross sections incorporating the evaporation effects are then given by

$$
\sigma_{N, Z}(E)=2 \pi \int_{b_{\min }}^{\infty} b \sum_{n, z} P_{N+n, Z+z}(E, b) P_{N+n, Z+z}^{n, z}\left[E_{N+n, Z+z}^{*}(E, b)\right] d b
$$

where the summation over $n$ and $z$ is taken for all possible numbers of evaporated nucleons. Excitation energy $E_{N+n, Z+z}^{*}(E, b)$ is calculated by employing Eq. (18).

\subsection{Numerical details}

We have developed our own TDHF code for heavy ion reactions [33]. In the code, singleparticle wave functions are represented on a uniform grid in three-dimensional Cartesian coordinates without any symmetry restrictions. We set the mesh spacing to be $0.8 \mathrm{fm}$. We use 11-point finite-difference formula for the first and second derivatives. For time evolution calculations, we utilize the 4th-order Taylor expansion method in operating the time propagation operator. Coulomb potential is calculated employing the Hockney's method [34] where Fourier transformations are performed in a box with the side length two times larger than the simulation box to obtain the potential of isolated boundary condition. To prepare 

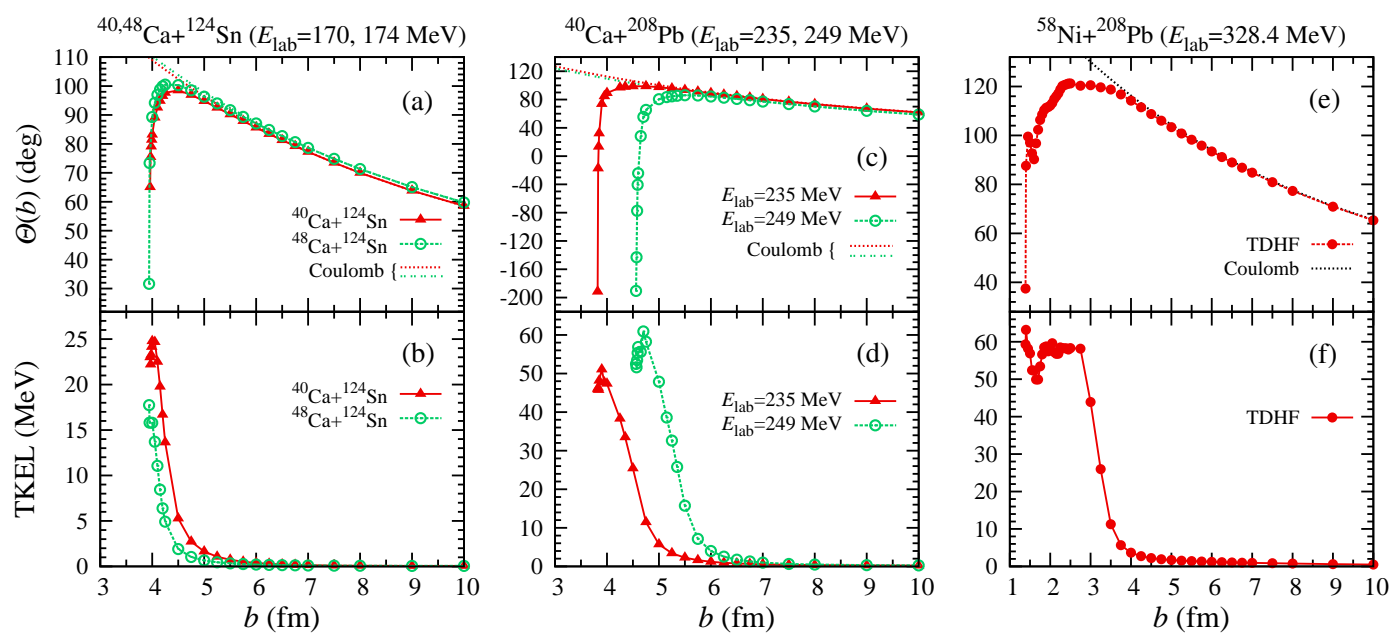

Figure 1: Deflection angle and total kinetic energy loss as functions of the impact paramëter for ${ }^{40,48} \mathrm{Ca}+{ }^{124} \mathrm{Sn}(\mathrm{a}, \mathrm{b}),{ }^{40} \mathrm{Ca}+{ }^{208} \mathrm{~Pb}(\mathrm{c}, \mathrm{d}),{ }^{58} \mathrm{Ni}+{ }^{208} \mathrm{~Pb}$ (e, f). Data were taken from Ref. [25].

the ground state, we used $26 \times 26 \times 26$ grid points. For the time evolution calculations, we used $60 \times 60 \times 26$ grid points. For the PNP calculation, we use the trapezoidal rule with $M$ discrete points. We set $M=200$ for all calculations presented below. Calculations are carried out for impact parameter region up to $10 \mathrm{fm}$.

\section{Results}

We use the Skyrme energy density functional, SLy5 parameter set. To obtain ground states, we first perform constrained Hartree-Fock calculations with deformation parameters $\beta=0,0.1$, and 0.2 , and $\gamma=0^{\circ}, 30^{\circ}$, and $60^{\circ}$. We then remove these constraints and re-minimize the energy. Among solutions, we regard the minimum energy solution as the Hartree-Fock ground state. The ground states of ${ }^{40,48} \mathrm{Ca}$ and ${ }^{208} \mathrm{~Pb}$ are spherical, while ${ }^{124} \mathrm{Sn}$ and ${ }^{58} \mathrm{Ni}$ are found to be oblate and prolate shapes, respectively, with $\beta \sim 0.11$ for both systems. In collision calculations, the symmetry axis of these deformed nuclei is set perpendicular to the reaction plane of our TDHF calculations. In Ref. [25], we showed that reaction processes do not depend much on the direction of the deformation for ${ }^{40} \mathrm{Ca}+{ }^{124} \mathrm{Sn}$ reaction.

The relative motion between the PLF and the TLF after the collision is characterized by the deflection angle $\Theta$ and the total kinetic energy loss (TKEL). They are calculated in the following way. At a certain time $t=t_{\mathrm{f}}$ after the collision, we consider two spheres around the center-of-mass of two reaction products, $\mathbf{R}_{\tau}\left(t_{\mathrm{f}}\right)(\tau=A$ or $B)$, with a radius $10 \mathrm{fm}$ for the PLF and $14 \mathrm{fm}$ for the TLF. Integrating densities of neutrons and protons inside the spheres, we evaluate the average number of nucleons, $A_{\tau}\left(t_{\mathrm{f}}\right)$, and protons $Z_{\tau}\left(t_{\mathrm{f}}\right)$. The reduced mass of the reaction products is given by $\mu\left(t_{\mathrm{f}}\right)=M_{A}\left(t_{\mathrm{f}}\right) M_{B}\left(t_{\mathrm{f}}\right) /\left(M_{A}\left(t_{\mathrm{f}}\right)+M_{B}\left(t_{\mathrm{f}}\right)\right)$, where $M_{\tau}=A_{\tau} m$ and $m$ is the nucleon mass. Using the relative vector $\mathbf{R}(t)=\mathbf{R}_{A}(t)-\mathbf{R}_{B}(t)$ and its time derivative $\dot{\mathbf{R}}(t)=(\mathbf{R}(t+\Delta t)-\mathbf{R}(t-\Delta t)) /(2 \Delta t)$, we evaluate the total kinetic energy (TKE) of outgoing fragments at infinity which is given by,

$$
\mathrm{TKE}=\frac{1}{2} \mu\left(t_{\mathrm{f}}\right) \dot{\mathbf{R}}^{2}\left(t_{\mathrm{f}}\right)+\frac{Z_{A}\left(t_{\mathrm{f}}\right) Z_{B}\left(t_{\mathrm{f}}\right) e^{2}}{\left|\mathbf{R}\left(t_{\mathrm{f}}\right)\right|} .
$$

The TKEL is given by TKEL $=E_{\text {rel }}-$ TKE, where $E_{\text {rel }}$ is the incident relative energy. The deflection angle $\Theta$ can be extracted from $\mathbf{R}\left(t_{\mathrm{f}}\right)$ and $\dot{\mathbf{R}}\left(t_{\mathrm{f}}\right)$ assuming that relative motion continues along the Coulomb trajectory for $t>t_{\mathrm{f}}$. 


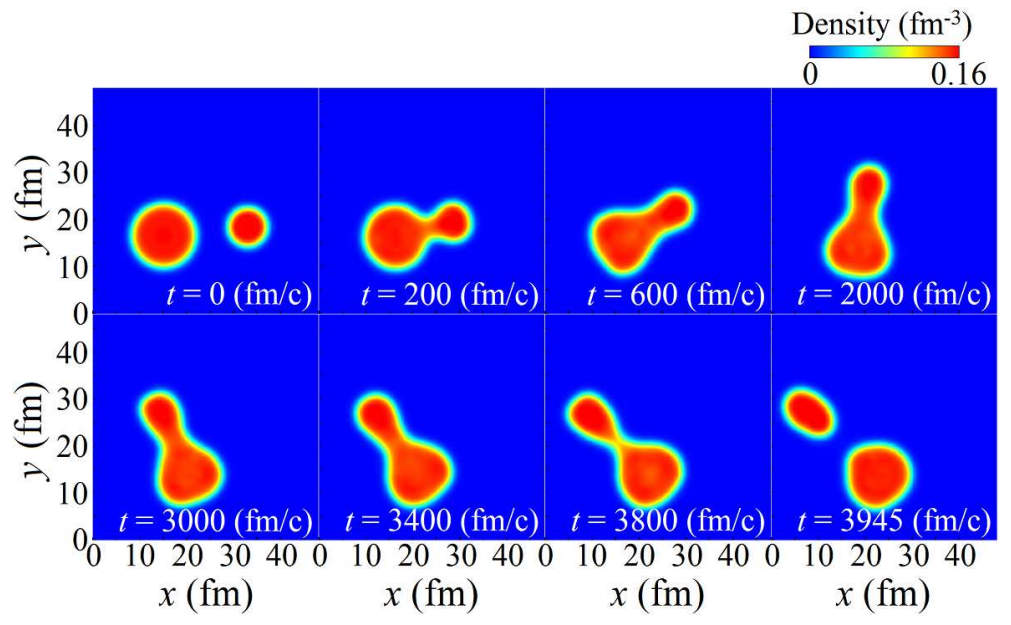

Figure 2: Snapshots of the density on the reaction plane for ${ }^{58} \mathrm{Ni}+{ }^{208} \mathrm{~Pb}$ reaction at $E_{\text {lab }}=328.4 \mathrm{MeV}$ and $b=1.39 \mathrm{fm}$. The figure was taken from Ref. [25].

In Fig. 1, we show deflection angle and TKEL for ${ }^{40,48} \mathrm{Ca}+{ }^{124} \mathrm{Sn}$ (a, b), ${ }^{40} \mathrm{Ca}+{ }^{208} \mathrm{~Pb}$ (c, d), and ${ }^{58} \mathrm{Ni}+{ }^{208} \mathrm{~Pb}(\mathrm{e}, \mathrm{f})$, as functions of the impact parameter. When the impact parameter is sufficiently large, the deflection angle coincides with that of the Coulomb trajectory. As the impact parameter decreases, the deflection angle becomes smaller because of the nuclear attractive interaction. As shown in Ref. [25], the Coulomb rainbow angles agree reasonably with a peak position of measured angular distributions.

The TKEL increases as the impact parameter decreases for all systems, as the deflection angle starts to deviate from the Coulomb trajectory. The maximum values reach $25 \mathrm{MeV}$ and $18 \mathrm{MeV}$ for ${ }^{40,48} \mathrm{Ca}+{ }^{124} \mathrm{Sn}, 50 \mathrm{MeV}$ and $60 \mathrm{MeV}$ for ${ }^{40} \mathrm{Ca}+{ }^{208} \mathrm{~Pb}$ at $E_{\text {lab }}=235 \mathrm{MeV}$ and $249 \mathrm{MeV}$, and $60 \mathrm{MeV}$ for ${ }^{58} \mathrm{Ni}+{ }^{208} \mathrm{~Pb}$. These values are correlated with the charge product of projectile and target nuclei, $Z_{\mathrm{P}} Z_{\mathrm{T}}$. The relatively small values in ${ }^{40,48} \mathrm{Ca}+{ }^{124} \mathrm{Sn}$ reflect that they are easily fused when two nuclei touch even slightly. As the charge product $Z_{\mathrm{P}} Z_{\mathrm{T}}$ increases, fusion suppression takes place. At a small impact parameter region, two nuclei collide more deeply and form a thick neck structure. Since two nuclei depart after forming the thick neck, produced fragments are highly excited and the TKEL becomes large for ${ }^{40} \mathrm{Ca},{ }^{58} \mathrm{Ni}+{ }^{208} \mathrm{~Pb}$ reactions. We note that the TKEL is almost constant for a certain range of impact parameter for ${ }^{58} \mathrm{Ni}+{ }^{208} \mathrm{~Pb}$, indicating a full momentum transfer in this system. In Fig. 2, we show snapshots of the density for the ${ }^{58} \mathrm{Ni}+{ }^{208} \mathrm{~Pb}$ reaction at $E_{\text {lab }}=328.4 \mathrm{MeV}$ and $b=1.39 \mathrm{fm}$. In this reaction, a formation of thick neck is seen during the reaction.

Figure 3 shows average number of transferred nucleons and $N / Z$ ratio as functions of impact parameter for four systems, ${ }^{40} \mathrm{Ca}+{ }^{124} \mathrm{Sn}$ (a, b), ${ }^{48} \mathrm{Ca}+{ }^{124} \mathrm{Sn}$ (c, d), ${ }^{40} \mathrm{Ca}+{ }^{208} \mathrm{~Pb}$ $(\mathrm{e}, \mathrm{f})$, and ${ }^{58} \mathrm{Ni}+{ }^{208} \mathrm{~Pb}(\mathrm{~g}, \mathrm{~h})$. In panels $(\mathrm{a}, \mathrm{c}, \mathrm{e}, \mathrm{g})$, a plus sign indicates that nucleons are transferred from target to projectile, while a minus sign indicates opposite. As seen from the panels, we find that neutrons are always transferred from the target to the projectile. Protons are transferred opposite, from the projectile to the target at large impact parameter region. However, the direction of the proton transfer changes at small impact parameter region.

From panels $(\mathrm{b}, \mathrm{d}, \mathrm{f}, \mathrm{h})$, we find that transfers proceed toward the $N / Z$ equilibrium between the projectile and the target nuclei. Since the ${ }^{48} \mathrm{Ca}+{ }^{124} \mathrm{Sn}$ is very close to the $N / Z$ equilibrium from the beginning, a very small number of nucleons are transferred on average as seen in Fig. 3 (c). Other systems, $\left({ }^{40} \mathrm{Ca}+{ }^{124} \mathrm{Sn}\right.$ and $\left.{ }^{40} \mathrm{Ca},{ }^{58} \mathrm{Ni}+{ }^{208} \mathrm{~Pb}\right)$, have a relatively large $N / Z$ asymmetry. The direction of the nucleon transfer except for protons at small impact parameter is consistent with the direction expected from the initial $N / Z$ ratios of the projectile and the target nuclei.

As the charge product $Z_{\mathrm{P}} Z_{\mathrm{T}}$ increases, binary fragments are produced even at a small impact parameter, because of the suppressed fusion reaction. In such collisions, we find a 

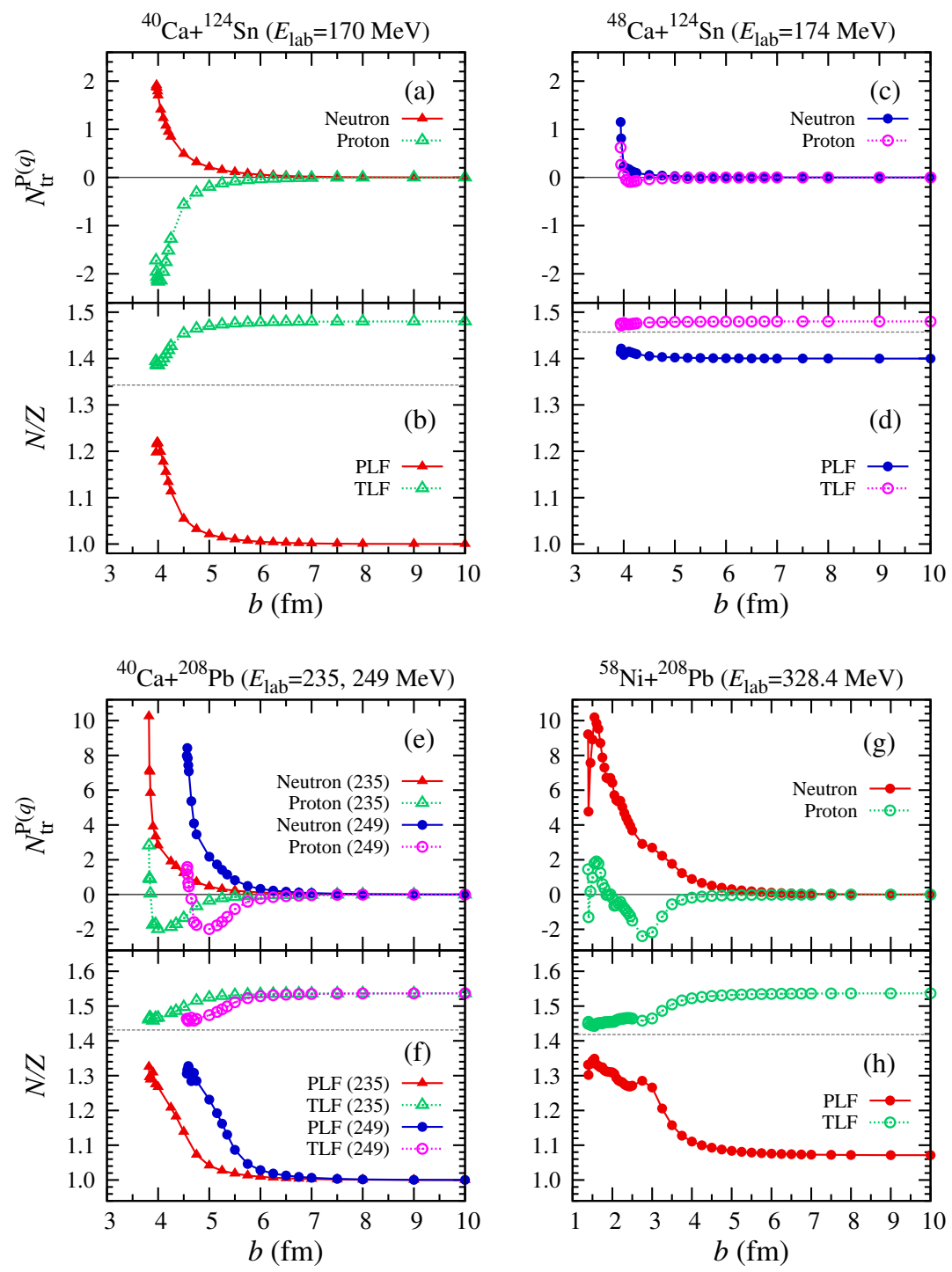

Figure 3: Average number of transferred nucleons and $N / Z$ ratios of PLF and TLF are shown as functions of the impact parameter for ${ }^{40} \mathrm{Ca}+{ }^{124} \mathrm{Sn}$ (a, b), ${ }^{48} \mathrm{Ca}+{ }^{124} \mathrm{Sn}$ (c, d), ${ }^{40} \mathrm{Ca}+{ }^{208} \mathrm{~Pb}(\mathrm{e}, \mathrm{f}),{ }^{58} \mathrm{Ni}+{ }^{208} \mathrm{~Pb}(\mathrm{~g}, \mathrm{~h})$. Data were taken from Ref. [25].

qualitative change in transfer mechanisms. In ${ }^{40} \mathrm{Ca},{ }^{58} \mathrm{Ni}+{ }^{208} \mathrm{~Pb}$ reactions, the number of transferred neutrons increases monotonically, while the number of transferred protons first increases and then decreases. At a certain small impact parameter, the number of transferred protons changes its sign. At a very small impact parameter region, both neutrons and protons are transferred toward the same direction, from target to projectile. This change in the transfer behavior is related to the thick neck formation. When the neck is broken, nucleons in the neck region are absorbed by one of the fragments. Because the neck is composed of both neutrons and protons, the absorption of the nucleons in the neck results in transfer of neutrons and protons in the same direction.

Using the PNP method, we obtain probability for each transfer channel as a function of the impact parameter from the TDHF wave functions after collision. As an example, we show transfer probabilities for ${ }^{58} \mathrm{Ni}+{ }^{208} \mathrm{~Pb}$ reaction in Fig. 4. Panels (a) and (b) show neutron transfer probabilities, while panels (c) and (d) show proton transfer probabilities. The shaded region indicates an impact parameter region in which fusion reactions take place. We note that the right panels (b) and (d) show results at a small impact parameter region. 

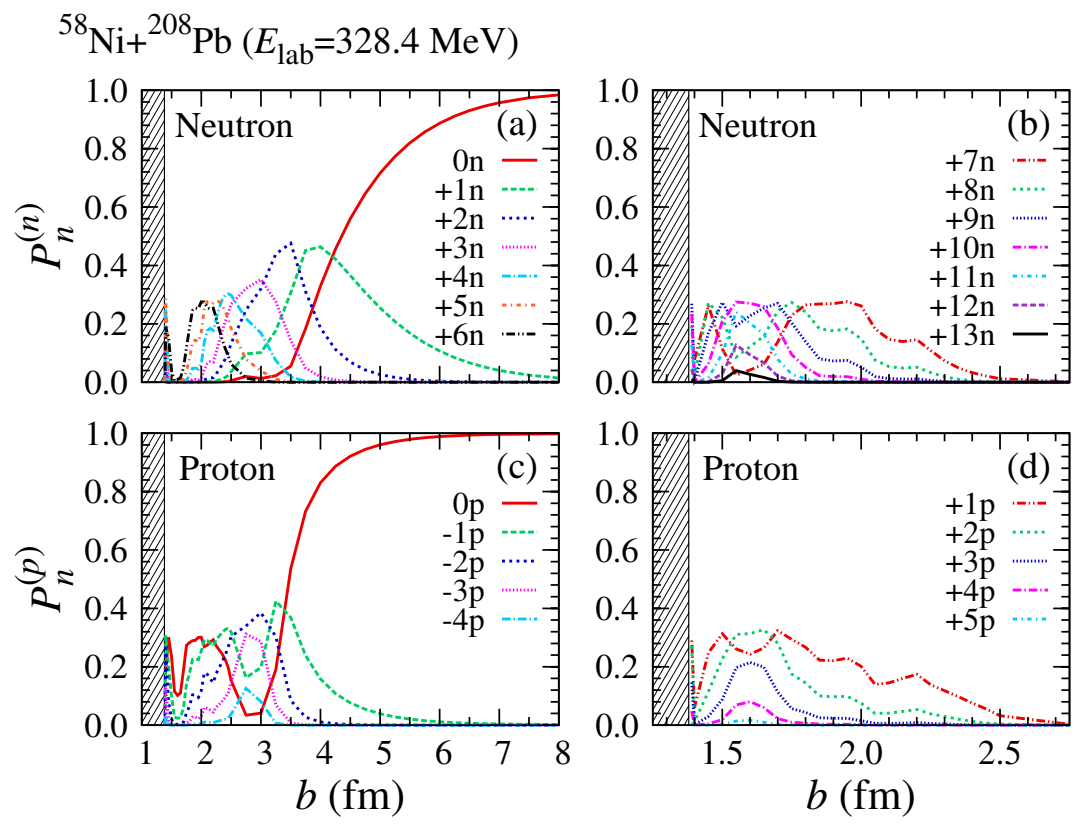

Figure 4: Transfer probabilities as functions of the impact parameter for ${ }^{58} \mathrm{Ni}+{ }^{208} \mathrm{~Pb}$ reaction at $E_{\mathrm{lab}}=328.4 \mathrm{MeV}$. The figure was taken from Ref. [25].

As seen from the figure, one-neutron transfer probability $(+1 \mathrm{n}$, green dashed line in (a)) has a long tail up to $8 \mathrm{fm}$. One-proton transfer probability $(-1 \mathrm{p}$, green dashed line in (c)) shows somewhat shorter tail compared with that of neutron. As the impact parameter decreases, transfer probabilities for various transfer channels become appreciable. The $+x \mathrm{n}$ in (a) indicates that $x$ neutrons are transferred from ${ }^{208} \mathrm{~Pb}$ to ${ }^{58} \mathrm{Ni}$. The $-x \mathrm{p}$ in (c) indicates that $x$ protons are transferred from ${ }^{58} \mathrm{Ni}$ to ${ }^{208} \mathrm{~Pb}$. The probabilities of neutron transfer in opposite direction are very small and are not shown. At a impact parameter region smaller than $3 \mathrm{fm}$ shown in panels (b) and (d), we find probabilities of many-neutron transfer from ${ }^{208} \mathrm{~Pb}$ to ${ }^{58} \mathrm{Ni}$, up to transfer of 13 neutrons. For protons, we find probabilities of transfer of up to 5 protons from ${ }^{208} \mathrm{~Pb}$ to ${ }^{58} \mathrm{Ni}$. The direction of transfer of neutrons and protons are consistent with the observation in Fig. 3 (g). Namely, neutrons and protons are transferred in opposite directions at a large impact parameter region, toward the charge equilibrium of the system. At a small impact parameter region, neutrons and protons transfer toward the same direction.

In Figs. 5 to 9, we show MNT cross sections, ${ }^{40} \mathrm{Ca}+{ }^{124} \mathrm{Sn}$ at $E_{\mathrm{lab}}=170 \mathrm{MeV}$ in Fig. 5 , ${ }^{48} \mathrm{Ca}+{ }^{124} \mathrm{Sn}$ at $E_{\text {lab }}=174 \mathrm{MeV}$ in Fig. $6,{ }^{40} \mathrm{Ca}+{ }^{208} \mathrm{~Pb}$ at $E_{\text {lab }}=235 \mathrm{MeV}$ and $249 \mathrm{MeV}$ in Figs. 7 and 8 , and ${ }^{58} \mathrm{Ni}+{ }^{208} \mathrm{~Pb}$ at $E_{\mathrm{lab}}=328.4 \mathrm{MeV}$ in Fig. 9. In all Figures, red points show experimental data, red-solid lines show calculated cross sections without the evaporation effects, and blue-dotted lines show calculated cross sections with the evaporation effects. We first compare calculated cross sections before taking account of the evaporation effects, given by red-solid lines, with measured cross sections. The cross sections for channels with small number of transferred nucleons are reasonably reproduced by the calculation for all systems. Except for ${ }^{48} \mathrm{Ca}+{ }^{124} \mathrm{Sn}$ reaction where $N / Z$ ratio is similar between the projectile and the target, cross sections are dominated by the transfer of protons from lighter to heavier nuclei. As the number of transferred protons increases, measured cross sections as a function of the neutron number of the PLF show peaks at less than that of the projectile. This indicates that both protons and neutrons are transferred in the same direction. However, in TDHF calculations without taking account of evaporation effects, the peak position locates at the neutron number more than that of the projectile. As a possible origin of the discrepancy, we mentioned the effect of evaporation from excited fragments [25].

The cross sections taking account of evaporation effects are shown by blue-dotted lines. 


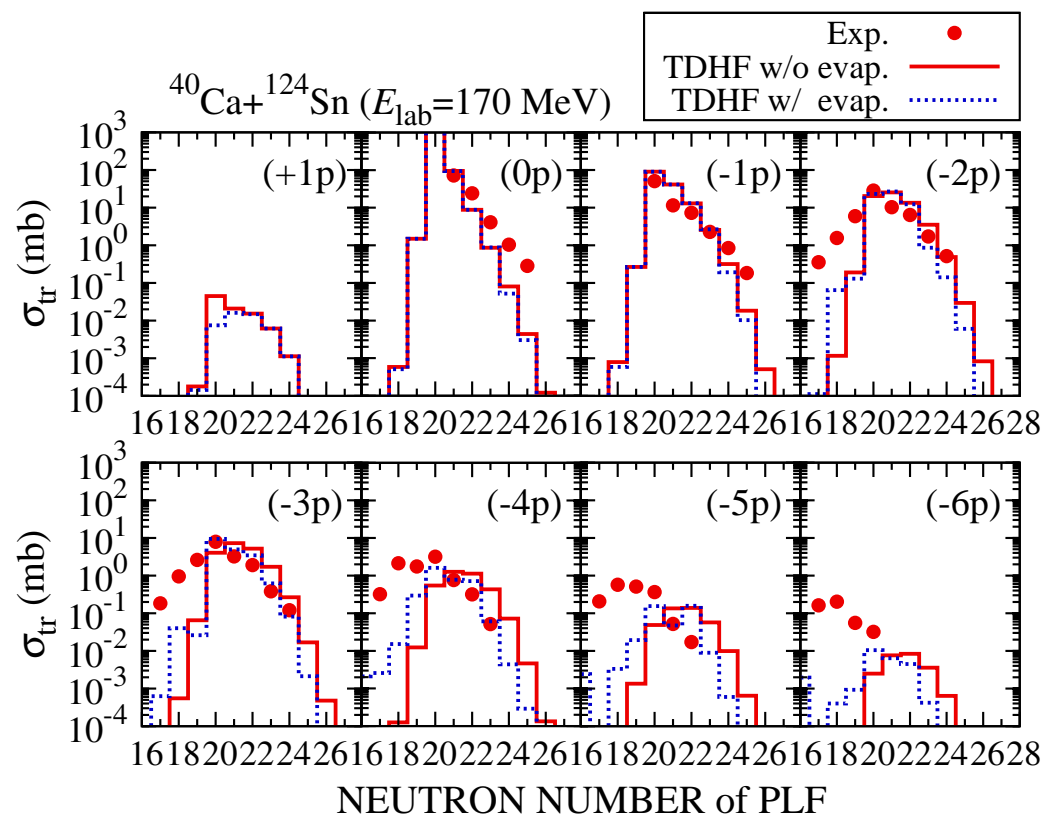

Figure 5: Production cross sections for PLF in ${ }^{40} \mathrm{Ca}+{ }^{124} \mathrm{Sn}$ reaction at $E_{\text {lab }}=170 \mathrm{MeV}$. The cross sections are classified according to the number of transferred protons which is indicated by $( \pm x \mathrm{p})$. The horizontal axis is the number of neutrons in the PLF. Red filled circles denote experimental data. Red solid lines (blue dotted lines) show the results of TDHF calculations without (with) effects of particle evaporation.

As seen from figures, the discrepancy is improved to some extent. However, the shift by the evaporation effect is not sufficient to fully remove the discrepancy. In other approaches such as the GRAZING, there remain discrepancies even after the evaporation effects are included, consistent with the present results. We consider the discrepancy suggests significance of the correlation effects that require description beyond the TDHF theory. We note that recently the same analysis was performed for an asymmetric system, ${ }^{18} \mathrm{O}+{ }^{206} \mathrm{~Pb}$, at an above barrier energy, which shows similar discrepancy [35].

The limitation of accuracy of the TDHF calculations comes from the fact that, for a collision specified by the impact parameter and the energy, there is only one mean-field that is used to evolve the system. From Fig. 3, we see that we have trajectories in which at most two protons are transferred $(-2 \mathrm{p})$, on average, from the projectile to the target. From

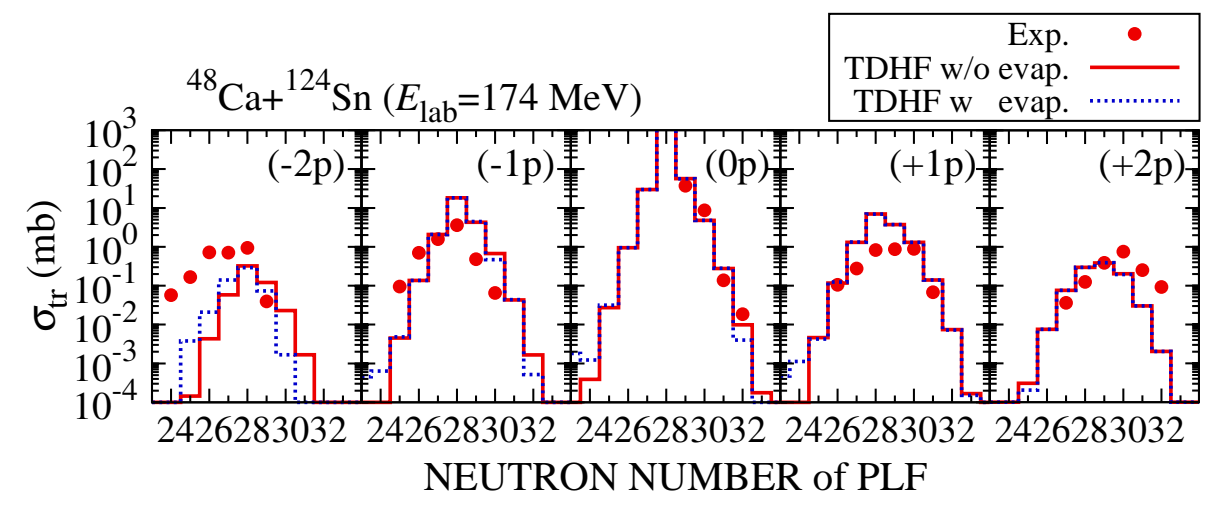

Figure 6: Same as Fig. 5, but for ${ }^{48} \mathrm{Ca}+{ }^{124} \mathrm{Sn}$ reaction at $E_{\text {lab }}=174 \mathrm{MeV}$. 


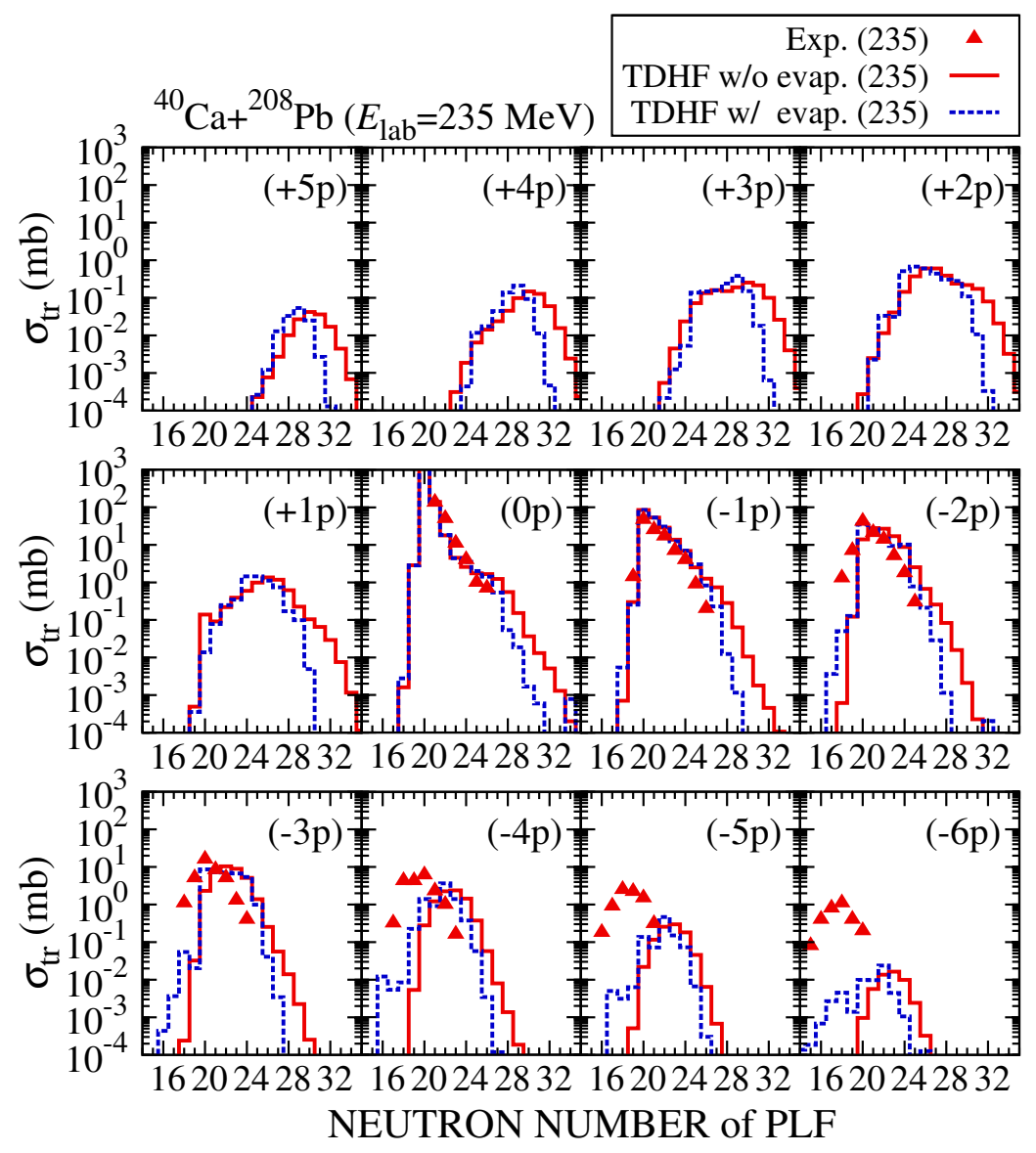

Figure 7: Same as Figs. 5 and 6 , but for ${ }^{40} \mathrm{Ca}+{ }^{208} \mathrm{~Pb}$ reaction at $E_{\text {lab }}=235 \mathrm{MeV}$.

these solutions, we extract probabilities accompanying up to 6 protons $(-6 \mathrm{p})$ by the PNP method. When the average number of transferred protons is about two from the projectile to the target, we find about two neutrons are transferred $(+2 n)$ from the target to the projectile. Because of this, our calculation results in the cross sections which show a peak at the two neutron transfer $(+2 \mathrm{n})$, irrespective of the number of proton transfers. To further improve the description, we need to employ the theory which goes beyond the TDHF theory, such as the method of Balian-Vénéroni [36, 37], the stochastic mean-field [38, 39, 40, 41, 42, 43] model, and the time-dependent generator coordinate method (TDGCM) [44, 45].

\section{Summary and prospect}

In this chapter, we reviewed our recent attempt to investigate multinucleon transfer (MNT) processes by the time-dependent Hartree-Fock (TDHF) theory combined with the particle-number projection (PNP) method. The TDHF theory has been successful to investigate nuclear many-body dynamics. Owing to a continuous development of computational facilities, it becomes possible to conduct systematic TDHF calculations for various systems with different initial conditions. The PNP method enables us to analyze the TDHF wave function after collision, providing reaction probabilities for a specific channel specified by the number of nucleons involved in the fragments.

As typical examples, we showed analyses of MNT processes in ${ }^{40,48} \mathrm{Ca}+{ }^{124} \mathrm{Sn},{ }^{40} \mathrm{Ca}+{ }^{208} \mathrm{~Pb}$, and ${ }^{58} \mathrm{Ni}+{ }^{208} \mathrm{~Pb}$ reactions for which precise experimental data are available. For all systems examined, we have found that nucleons are transferred toward the direction of the charge equilibrium. For systems with a large difference of $N / Z$ ratio between the projectile and 


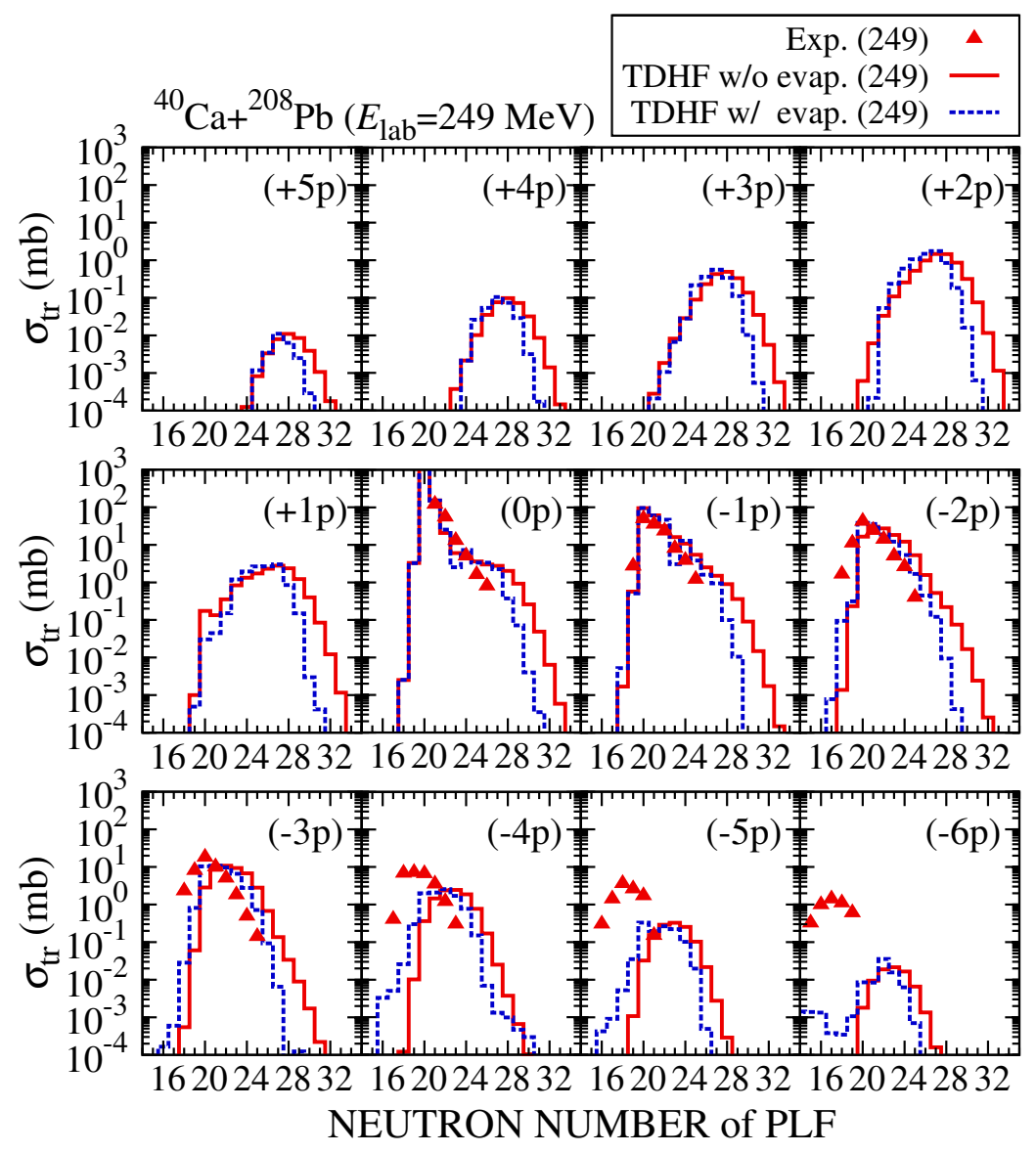

Figure 8: Same as Figs. 5, 6, and 7, but for ${ }^{40} \mathrm{Ca}+{ }^{208} \mathrm{~Pb}$ reaction at $E_{\text {lab }}=249 \mathrm{MeV}$.

the target, $\left({ }^{40} \mathrm{Ca}+{ }^{124} \mathrm{Sn},{ }^{40} \mathrm{Ca}+{ }^{208} \mathrm{~Pb}\right.$, and $\left.{ }^{58} \mathrm{Ni}+{ }^{208} \mathrm{~Pb}\right)$, transfers of a number of nucleons are observed in the calculation. For a system with a small $N / Z$ asymmetries, ${ }^{48} \mathrm{Ca}+{ }^{124} \mathrm{Sn}$, nucleons do not transfer much on average.

As the charge product of the projectile and the target nuclei, $Z_{\mathrm{P}} Z_{\mathrm{T}}$, increases, we found that the two nuclei are once connected by forming a thick neck and then reseparate. When the thick neck ruptures, nucleons inside the neck are absorbed by one of the fragments, resulting in transfers of many neutrons and protons toward the same direction. We consider that this neck breaking process is a precursor of quasifission process which dominates in small impact parameter collisions between heavy nuclei.

For each transfer channel specified by the number of neutrons and protons included in reaction products, we evaluate transfer probabilities and excitation energies of the reaction products by the PNP method. We also evaluate evaporation probabilities of excited fragments employing a statistical model. Using these quantities, we calculate production cross sections of each reaction product including effects of particle evaporation. The obtained cross sections are compared with measurements.

From the comparisons, we have found that the TDHF theory nicely reproduces measured cross sections when the number of transferred nucleons is small. As the number of transferred nucleons increases, however, we have found that the accuracy of the TDHF theory to reproduce measured cross sections is reduced. Although the inclusion of the evaporation effects has improved the agreement between experimental and calculated results, the calculations still substantially underestimate the cross sections when a number of nucleons are transferred. This failure indicates a necessity of improving the theoretical framework.

We are now extending our study toward quasifission-induced MNT processes in a collision between heavy nuclei. In such reactions, disintegration processes of not only particle 


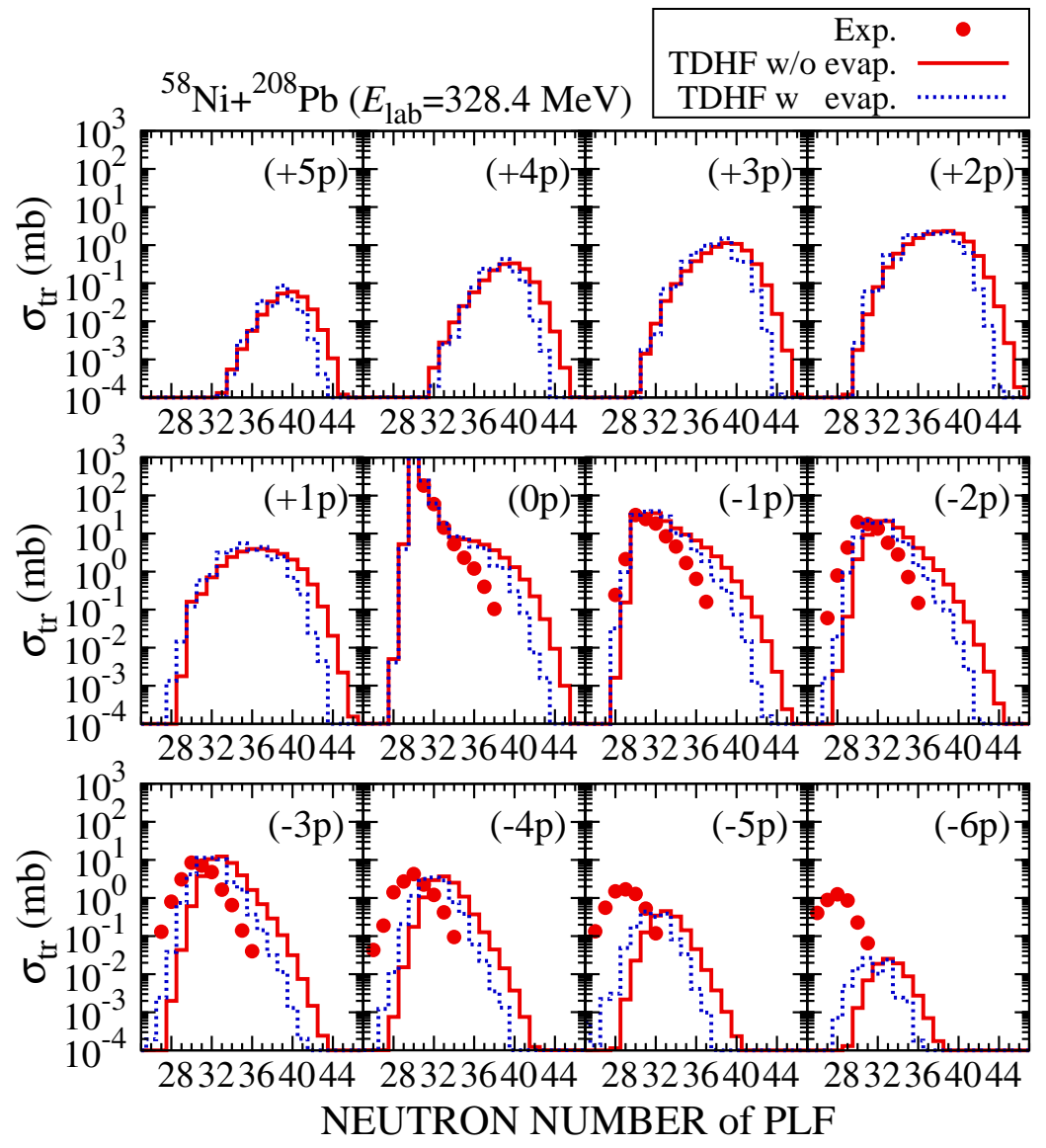

Figure 9: Same as Figs. 5, 6, 7, and 8, but for ${ }^{58} \mathrm{Ni}+{ }^{208} \mathrm{~Pb}$ reaction at $E_{\text {lab }}=328.4 \mathrm{MeV}$.

evaporation but also transfer-induced fissions should be considered. Since the PNP method allows us to evaluate expectation values of any observables of reaction products for each transfer channel, we plan to use them in sophisticated statistical models, such as PACE, CASCADE, GEMINI, or HIVAP, to evaluate effects of secondary processes on measurable cross sections.

Finally we would like to mention possible directions to improve theoretical descriptions of MNT processes. A well-known limitation of the TDHF calculation is the fact that it employs a single mean-field in the time evolution calculation for a given initial condition, although we extract reaction probabilities of different numbers in the fragments from the solution. To include the difference of the mean-field for transfer channels, the time-dependent generator coordinate method (TDGCM) [44, 45] will be useful. Although practical applications of the TDGCM to heavy ion reactions are challenging, it should be a promising extension for MNT reactions. We note that the pairing correlation is not taken into account in the present calculation employing the TDHF theory. The time-dependent Hartree-FockBogoliubov (TDHFB) theory will definitely provide useful information to this direction $[46,47,48,49,50,51]$.

Recently, a gamma-ray spectroscopic study of reaction products of MNT reactions has become feasible $[52,53]$. Although we have only achieved number projection in the present work, our method can be extended to include parity and angular momentum projections [54]. Calculations and making comparison with experimental data will provide us rich information on microscopic reaction mechanisms. 


\section{Acknowledgments}

We would like to appreciate Prof. J.A. Maruhn for his prominent contributions to nuclear physics society, especially for continuous studies using the time-dependent Hartree-Fock theory. This research work used computational resources of the HPCI system provided by Information Initiative Center, Hokkaido University, through the HPCI System Research Project (Project IDs: hp120204, hp140010, and hp150010). This work was supported by the Japan Society for the Promotion of Science (JSPS) Grant-in-Aid for JSPS Fellows, Grant Number 25-241.

\section{References}

[1] C.H. Dasso, G. Pollarolo, and A. Winther, Phys. Rev. Lett. 73, 1907 (1994).

[2] C.H. Dasso, G. Pollarolo, and A. Winther, Phys. Rev. C 52, 2264 (1995).

[3] C. Simenel, Eur. Phys. J. A 48, 152 (2012).

[4] V.I. Zagrebaev and W. Greiner, Phys. Rev. C 87, 034608 (2013).

[5] V.I. Zagrebaev, B. Fornal, S. Leoni, and W. Greiner, Phys. Rev. C 89, 054608 (2014).

[6] L. Zhu, Z.-Q. Feng, and F.-S. Zhang, arXiv.1503.04421 [nucl-th].

[7] R. Yanez and W. Loveland, Phys. Rev. C 91, 044608 (2015).

[8] V. I. Zagrebaev and W. Greiner, Phys. Rev. C 83, 044618 (2011).

[9] Y.X. Watanabe, Y.H. Kim, S.C. Jeong, Y. Hirayama, N. Imai, H. Ishiyama, H.S. Jung, H. Miyatake, S. Choi, J.S. Song, E. Clement, G. de France, A. Navin, M. Rejmund, C. Schmitt, G. Pollarolo, L. Corradi, E. Fioretto, D. Montanari, M. Niikura, D. Suzuki, H. Nishibata, and J. Takatsu, Phys. Rev. Lett. 115, 172503 (2015).

[10] A. Winther, Nucl. Phys. A572, 191 (1994); A594, 203 (1995).

[11] E. Vigezzi and A. Winther, Ann. Phys. (N.Y.) 192, 432 (1989).

[12] V. Zagrebaev and W. Greiner, J. Phys. G: Nucl. Part. Phys. 31, 825 (2005).

[13] V. Zagrebaev and W. Greiner, J. Phys. G: Nucl. Part. Phys. 34, 1 (2007).

[14] N.V. Antonenko, E.A. Cherepanov, A.K. Nasirov, V.P. Permjakov, and V.V. Volkov, Phys. Rev. C 51, 2635 (1995).

[15] G.G Adamian, N.V. Antonenko, and W. Scheid, Nucl. Phys. A618, 176 (1997).

[16] G.G Adamian, N.V. Antonenko, W. Scheid, and V.V. Volkov, Nucl. Phys. A627, 361 (1997).

[17] G.G Adamian, N.V. Antonenko, W. Scheid, and V.V. Volkov, Nucl. Phys. A633, 409 (1998).

[18] L. Corradi, G. Pollarolo, and S. Szilner, J. Phys. G: Nucl. Part. Phys. 36, 113101 (2009).

[19] J.S. Barrett, W. Loveland, R. Yanez, S. Zhu, A.D. Ayangeakaa, M.P. Carpenter, J.P. Greene, R.V.F. Janssens, T. Lauritsen, E.A. McCutchan, A.A. Sonzogni, C.J. Chiara, J.L. Harker, and W.B. Walters, Phys. Rev. C 91, 064615 (2015).

[20] J.W. Negele, Rev. Mod. Phys. 54, 913 (1982).

[21] S.E. Koonin, K.T.R. Davies, V. Maruhn-Rezwani, H. Feldmeier, S.J. Krieger, and J.W. Negele, Phys. Rev. C 15, 1359 (1977). 
[22] H. J. Lüdde and R. M. Dreizler, J. Phys. B 16, 3973 (1983).

[23] R. Nagano, K. Yabana, T. Tazawa, and Y. Abe, Phys. Rev. A 62, 062721 (2000).

[24] C. Simenel, Phys. Rev. Lett. 105, 192701 (2010).

[25] K. Sekizawa and K. Yabana, Phys. Rev. C 88, 014614 (2013).

[26] L. Corradi, J. H. He, D. Ackermann, A. M. Stefanini, A. Pisent, S. Beghini, G. Montagnoli, F. Scarlassara, G. F. Segato, G. Pollarolo, C. H. Dasso, and A. Winther, Phys. Rev. C 54, 201 (1996).

[27] L. Corradi, A. M. Stefanini, J. H. He, S. Beghini, G. Montagnoli, F. Scarlassara, G. F. Segato, G. Pollarolo, and C. H. Dasso, Phys. Rev. C 56, 938 (1997).

[28] L. Corradi, A. M. Vinodkumar, A. M. Stefanini, E. Fioretto, G. Prete, S. Beghini, G. Montagnoli, F. Scarlassara, G. Pollarolo, F. Cerutti, and A. Winther, Phys. Rev. C 66, 024606 (2002).

[29] S. Szilner, L. Corradi, G. Pollarolo, S. Beghini, B. R. Behera, E. Fioretto, A. Gadea, F. Haas, A. Latina, G. Montagnoli, F. Scarlassara, A. M. Stefanini, M. Trotta, A. M. Vinodkumar, and Y. Wu, Phys. Rev. C 71, 044610 (2005).

[30] K. Sekizawa and K. Yabana, Phys. Rev. C 90, 064614 (2014).

[31] K. Sekizawa and K. Yabana, EPJ Web of Conferences 86, 00043 (2015).

[32] I. Dostrovsky and Z. Fraenkei, Phys. Rev. 116, 683 (1959); 119, 2098 (1960).

[33] K. Sekizawa, "Multinucleon Transfer Reactions and Quasifission Processes in TimeDependent Hartree-Fock Theory", Ph.D. thesis, University of Tsukuba, Tsukuba, Japan, 2015.

[34] J.W. Eastwood and D.R.K. Brownrigg, J. Compt. Phys. 32, 24 (1979).

[35] B.J. Roy, A. Parmar, U.K. Pal, H. Kumawat, V. Jha, S.K. Pandit, V.V. Parkar, K. Ramachandran, K. Mahata, A. Pal, S. Santra, A.K. Mohanty, and K. Sekizawa, Phys. Rev. C 92, 042603 (2015).

[36] R. Balian and M. Vénéroni, Phys. Rev. Lett. 47, 1353 (1981).

[37] C. Simenel, Phys. Rev. Lett. 106, 112502 (2011).

[38] S. Ayik, Phys. Lett. B 658, 174 (2008).

[39] K. Washiyama, S. Ayik, and D. Lacroix, Phys. Rev. C 80, 031602(R) (2009).

[40] S. Ayik, K. Washiyama, and D. Lacroix, Phys. Rev. C 79, 054606 (2009).

[41] D. Lacroix, S. Ayik, and B. Yilmaz, Phys. Rev. C 85, 041602(R) (2012).

[42] D. Lacroix, D. Gambacurta and S. Ayik, Phys. Rev. C 87, 061302(R) (2013).

[43] B. Yilmaz, S. Ayik, D. Lacroix, and O. Yilmaz, Phys. Rev. C 90, 024613 (2014).

[44] J.F. Berger, M. Girod, and D. Gogny, Nucl. Phys. A428, 23 (1984).

[45] C. Simenel, J. Phys. G: Nucl. Part. Phys. 41, 094007 (2014).

[46] Y. Hashimoto and K. Nodeki, arXiv:0707.3083 [nucl-th].

[47] B. Avez, C. Simenel, and Ph. Chomaz, Phys. Rev. C 78, 044318 (2008).

[48] I. Stetcu, A. Bulgac, P. Magierski, and K.J. Roche, Phys. Rev. C 84, 051309(R) (2011).

[49] Y. Hashimono, Eur. Phys. J. A 48, 55 (2012). 
[50] Y. Hashimoto, Phys. Rev. C 88, 034307 (2013).

[51] A. Bulgac, P. Magierski, K.J. Roche, I. Stetcu, arXiv:1511.00738 [nucl-th].

[52] S. Szilner, C.A. Ur, L. Corradi, N. Mărginean, G. Pollarolo, A.M. Stefanini, S. Beghini, B.R. Behera, E. Fioretto, A. Gadea, B. Guiot, A. Latina, P. Mason, G. Montagnoli, F. Scarlassara, M. Trotta, G. de Angelis, F. Della Vedova, E. Farnea, F. Haas, S. Lenzi, S. Lunardi, R. Mărginean, R. Menegazzo, D.R. Napoli, M. Nespolo, I.V. Pokrovsky, F. Recchia, M. Romoli, M.-D. Salsac, N. Soić, and J.J. Valiente-Dobón, Phys. Rev. C 76, 024604 (2007).

[53] A. Vogt, B. Birkenbach, P. Reiter, L. Corradi, T. Mijatović, D. Montanari, S. Szilner, D. Bazzacco, M. Bowry, A. Bracco, B. Bruyneel, F.C.L. Crespi, G. de Angelis, P. Désesquelles, J. Eberth, E. Farnea, E. Fioretto, A. Gadea, K. Geibel, A. Gengelbach, A. Giaz, A. Görgen, A. Gottardo, J. Grebosz, H. Hess, P.R. John, J. Jolie, D.S. Judson, A. Jungclaus, W. Korten, S. Leoni, S. Lunardi, R. Menegazzo, D. Mengoni, C. Michelagnoli, G. Montagnoli, D. Napoli, L. Pellegri, G. Pollarolo, A. Pullia, B. Quintana, F. Radeck, F. Recchia, D. Rosso, E. Şahin, M.D. Salsac, F. Scarlassara, P.-A. Söderström, A.M. Stefanini, T. Steinbach, O. Stezowski, B. Szpak, Ch. Theisen, C. Ur, J.J. Valiente-Dobón, V. Vandone, and A. Wiens, Phys. Rev. C 92, 024619 (2015).

[54] P. Ring and P. Schuck, "The Nuclear Many-Body Problem", (Springer-Verlag, 1980). 